\title{
Oportunidades y desafíos de la historia local: algunas reflexiones desde un campo en expansión
}

\author{
Andrea Andújar \\ andreaandujar@gmail.com \\ Universidad de Buenos Aires, Argentina \\ CONICET, Argentina \\ Leandro Lichtmajer \\ leandrolichtmajer@gmail.com \\ Facultad de Filosofía y Letras. Universidad Nacional de Tucumán, Argentina \\ CONICET, Argentina
}

Cita sugerida: Andújar, A. y Lichtmajer, L. (2021).

Oportunidades y desafíos de la historia local:

algunas reflexiones desde un campo en expansión.

Anuario del Instituto de Historia Argentina, 21(1),

e132. https://doi.org/10.24215/2314257Xe132

Las investigaciones en clave local vienen ganando terreno en la producción sobre la historia argentina del siglo XX. De manera sostenida, aunque sin la estridencia de otros desarrollos historiográficos, los ejercicios de análisis localizados fueron ensayados desde registros múltiples inscriptos en la historia social, política y económica. Esta naturaleza diversa desalienta los intentos por sistematizar una producción vasta y en expansión, aunque no impide reconocer algunos de sus trazos más fecundos. En ese reconocimiento es factible individualizar líneas de investigación que ofrecieron a las miradas localizadas un terreno fructífero para desplegar sus aportes conceptuales y metodológicos.

En el campo de la historia política cobraron relieve, por ejemplo, los estudios sobre los partidos en el período comprendido entre la reforma electoral de 1912 y el primer peronismo. Los interrogantes sobre las tramas locales de las organizaciones partidarias fueron relevantes en esa etapa, definida globalmente por 
el tránsito hacia la "política de masas" y las transformaciones en las formas de producción del sufragio, las campañas, el reclutamiento y la construcción de identidades partidarias. Desde la década de 1990 hasta la fecha, un bagaje diverso de estudios localizados sobre socialistas, comunistas, peronistas y, en menor medida, conservadores y radicales, enriqueció nuestra comprensión sobre dichos actores. Mediante un ejercicio de contrastación atento a las hipótesis "macro" -ajeno, por lo tanto, al "localismo estrecho" del que se nos suele alertar a la hora de emprender estos ejercicios analíticos (Massey, 1995; Terradas i Saborit, 2001; Pons y Serna, 2007) - la producción descentró las miradas de las esferas nacionales, el ámbito capitalino y la matriz urbana en la construcción de las interpretaciones, incorporando espacios y sujetos de análisis previamente soslayados.

Entre los aportes de las miradas localizadas puede señalarse su capacidad de reponer la estrecha interrelación entre los entramados partidarios, el espacio en el que desplegaron sus actividades y su entorno social, configurado por redes de relaciones que se proyectan sobre las actividades proselitistas y los ámbitos de sociabilidad (comités, centros, unidades básicas, etc.). Por otro lado, la porosidad de las fronteras entre los partidos fue recuperada a través de las trayectorias personales y las expresiones de una cultura política permeada por las improntas locales, así como por la relevancia de las prácticas informales en la construcción de los liderazgos, el reclutamiento y las disputas por el poder. Estas aristas permitieron repensar en qué medida las normas escritas y las directivas de las autoridades centrales influyeron en las experiencias concretas de dirigentes, militantes y simpatizantes, revelando una dinámica compleja y mutuamente relacionada. Al desentrañar prácticas ubicadas en los intersticios de las reglas formalizadas, ajenas a una mirada normativa del comportamiento político o las preferencias electorales, la producción recuperó la capacidad de agencia de sujetos ya contemplados a la par que visibilizó a colectivos no incluidos formalmente en la disputa electoral pero activamente involucrados en la vida política, tales como grupos de mujeres y organizaciones que involucraron a la niñez en las actividades proselitistas. Este ejercicio cuestionó, asimismo, el "deber ser" partidario proyectado en los estatutos, reglamentos y cartas orgánicas, relativizando el peso de las esferas centrales en las dinámicas locales y problematizando la relación entre las autoridades y los organismos de base. De ese modo, las miradas sobre los partidos en sede local tendieron a desmarcarse de los pares centralización/ descentralización o autonomía/heteronomía para configurar un conjunto dinámico de interrelaciones que revelaron sus márgenes de acción y negociación.

Otro campo fértil se detecta en la historia social, especialmente aquella interesada en el mundo del trabajo. Las investigaciones situadas en la escala local promovieron lecturas capaces de poner en valor procesos y relaciones sociales cuyas particularidades ofrecieron una interpretación más profunda y compleja de la historia de la clase trabajadora, a veces complementando los relatos más generales, otras veces contrariando sus puntos de partida y sus conclusiones. Un ejemplo de ello se encuentra en los estudios sobre comunidades obreras ligadas a diversas actividades económicas. La localización del punto de mira, emprendida con fuerza analítica entre finales del siglo pasado e inicios del actual, brindó mayor nitidez histórica a una amplia variedad de sujetos y experiencias, permitiendo reconocer la multiplicidad de iniciativas, intereses y conflictos presentes en su proceso formativo y en su decurso. Así, el examen de comunidades que giraron en torno a actividades ferroviarias, azucareras, textiles, frigoríficas, metalúrgicas o petroleras, emplazadas en una vastedad de regiones, logró ir más allá de las voluntades empresariales y la demarcación de las fronteras territoriales y sociales que esas voluntades dictaminaron (en función de los intereses patronales y de sus contradicciones o acuerdos con los poderes municipales, provinciales o nacionales), recalando en las acciones y expectativas de los y las trabajadores, en sus redes y relaciones de sociabilidad gestadas en su cotidianeidad laboral y social. Al advertirlos en una dinámica que excedió el tiempo y el lugar de trabajo, fue posible entender cómo esos varones y mujeres de la clase trabajadora edificaron sus propias organizaciones, demandas sindicales y horizontes políticos en función del espacio y el tiempo en que vivían pero, también, en conexión con contextos más amplios, con los que interactuaron de manera variada: por ejemplo, los tejidos en los clubes 
sociales y deportivos, las bibliotecas obreras y populares, los cines y teatros o durante los picnics y las fiestas al aire libre.

La revisión de esos vínculos, junto con el interés por relevar dimensiones menos perceptibles en miradas pretendidamente nacionales, informaron sobre cuestiones como la pervivencia de formas de ganarse la vida distintas a las comprendidas por el trabajo asalariado, el lugar de la migración como estrategia de supervivencia familiar en los años 1920 y 1930, o la complementariedad de diversas prácticas de organización obrera (como las sindicales y las asociaciones de ayuda mutua). Una parte importante de esta renovada agenda se focaliza en la cultura obrera, cuyo estudio revela una vastedad de iniciativas en tal sentido. Estas se ligaron a los sindicatos y las izquierdas en las primeras décadas del siglo XX pero recuperaron también las aristas de las disputas propiciadas en ese terreno por las derechas, actor que ganó en complejidad y heterogeneidad gracias a investigaciones cada vez más profusamente ubicadas en la escala local. Otro tanto puede señalarse sobre la densidad adquirida por el análisis de los procesos de radicalización de los años sesenta y setenta del siglo pasado así como en los trabajos dedicados a examinar las resistencias organizadas por los movimientos de trabajadoras y trabajadores desocupados ante la profundización del neoliberalismo en la pos-dictadura, tales como los cortes de ruta protagonizados por los movimientos piqueteros surgidos en la década de 1990. Asimismo, el estudio de las dinámicas del mundo del trabajo rural en sus diversas espacialidades permitió detectar relaciones laborales y tramas sociales más complejas, que escapan a generalizaciones simplificadoras.

En esa trama localizada, la historia de las mujeres y los estudios de géneros encontraron una vía eficaz para ahondar en la densidad de las interpretaciones sobre un pasado amplio, comprendido entre mediados del siglo XIX y la actualidad. Los aportes metodológicos de largo aliento - a través de la validación de un conjunto de fuentes de diversa factura- se conjugaron con la formulación de tematizaciones y problemáticas sobre el mundo de los y las trabajadores que abonaron la reinserción de la clase como categoría valiosa para el estudio histórico, pero necesariamente imbricada con el género. El examen sobre los procesos formativos de la fuerza de trabajo develó las formas en que ciertas nociones de masculinidad y feminidad proletaria terciaron en la delimitación de oficios, tareas y profesiones de mujeres y varones así como en las organizaciones y acciones de protesta obreras. Así, se pusieron en evidencia las diferenciales tasas y condiciones de explotación sufridas por mujeres y varones trabajadores, sopesando el impacto de las tareas de cuidado y reproducción de la familia obrera en ese aspecto.

Si en los estudios localizados las categorías de clase y género tomaron la delantera a la hora de componer una comprensión más compleja del decurso de la clase trabajadora, la imbricación con otras nociones definitorias de la opresión -como las de racialidad y etnicidad, por ejemplo- despuntan una presencia cada vez mayor, revelando experiencias que colaboran en desmontar una saga histórica construida en torno a la generalización de la experiencia -también idealizada - de los trabajadores adultos blancos, de origen europeo y urbanos. Esta apuesta analítica alentó, además, la ubicación del espacio capitalino y bonaerense como parte de la traza de lo local, empeño que reveló entramados barriales y comunitarios heterogéneos y dinámicos, enlazados a partir del trajinar de sujetos cuyas prácticas y relaciones habían permanecido ocultas. La asunción de una perspectiva localizada de análisis brindó la posibilidad, incluso, de forzar al centro a desnaturalizar prácticas, sentidos y creencias elevados a un carácter nacional, problematizando sus dinámicas sociales, barriales y comunitarias en función de un juego de escalas.

La breve reseña de estas líneas de trabajo muestra un proceso de maduración que vuelve factible inquirir por el estatus de la historia local como práctica historiográfica. En ese sentido, la densidad de investigaciones denota la ductilidad de estrategias metodológicas, abordajes teóricos y preguntas que proponen nuevos temas y problemas de investigación a la par que revisitan, con otras lentes, los ya indagados. Advertir esta entidad de la historia local anima a preguntar, también, por sus diferencias y similitudes con otras perspectivas que podrían resultar afines. Por ejemplo, la microhistoria, la historia "a ras del suelo" o, particularmente para el campo historiográfico argentino, aquella que se reconoce y se autodenomina como historia regional desde sus primeras formulaciones en la década de 1960. ¿Contienen la historia local y regional apuestas 
metodológicas, conceptuales y teóricas que ameritan un ejercicio de distinción? ¿ O pueden utilizarse como sinónimos? Como lo revelan los sostenidos esfuerzos reflexivos elaborados por diversos colegas, en el marco del extenso debate sobre las perspectivas regionales y locales en los estudios sobre historia argentina, ha sido habitual la tendencia a pensarlas de manera conjunta, aún cuando no se pierdan de vista sus especificidades. ${ }^{1}$ Esto no es azaroso y se asienta en un conjunto de bagajes compartidos, tales como el juego de escalas, la problematización del espacio como categoría de análisis, la reposición de la capacidad de agencia de los sujetos y la reacción contra una historiografía pretendidamente nacional. Sin embargo, entendemos que la posibilidad de delimitar los perímetros de lo local, tarea por definición inacabada, constituye un horizonte deseable a la hora de ponderar sus trazos previos e imaginar sus rumbos futuros en tanto estrategia metodológica y propuesta analítica. ${ }^{2}$

En virtud de estas consideraciones, el presente dossier reúne las miradas de tres reconocidas historiadoras que, desde diversos registros, aportan a la reflexión sobre los análisis localizados. Se combinan allí los resultados de investigaciones de largo aliento con la búsqueda de un repertorio propositivo, que permite imaginar recorridos y agendas posibles, así como algunos diálogos conceptuales y metodológicos de lo local.

En el texto que abre el dossier, titulado "Microhistoria, Microanálisis, Historia Regional, Historia Local”, Susana Bandieri invita a repensar el estado actual de la historia local y regional en la Argentina. Con ese fin traza un recorrido conceptual e historiográfico que incluye dos marcos de referencia centrales para ajustar el foco de observación: la microhistoria y el microanálisis. Al retomar el debate en torno a los puntos de contacto y diferencia entre lo micro y lo local, Bandieri aborda una dimensión cardinal para el objetivo que anima este dossier ya que es en la mutua relación y los puntos de disonancia entre las miradas locales y micro donde se definen algunas coordenadas y equívocos decisivos para asumir este tipo de abordajes. Su texto enmarca, así, una discusión cara a la producción historiográfica argentina y europea, que reconoce en la síntesis reciente de Ángelo Torre (2018) una valiosa contribución. La autora también desanda el camino seguido por la historia regional y local en nuestro país, reflexionando sobre sus mixturas, alcances y límites. Ello le permite subrayar sus elementos en común y recuperar los aportes de ambas perspectivas, sobrevolando una noción que trasciende la discusión historiográfica y metodológica para proyectarse hacia el terreno de la enseñanza de la historia. Bandieri se pregunta en qué medida las miradas locales y regionales, con sus potencialidades para problematizar periodizaciones, hipótesis o creencias arraigadas en el sentido común historiográfico, fueron efectivamente incorporadas en las pedagogías sobre el pasado. Si bien la respuesta a este interrogante se adentra en la historia de la Patagonia, espacio en el que la autora desplegó sus múltiples y fructíferas líneas de indagación, sus reflexiones involucran una alerta y un llamado a repensar esta problemática en y para otras geografías de la Argentina.

Sigue a este estudio un texto de Gabriela Águila que lleva por título "La dictadura, las formas de la represión y los estudios a escala local”. Este artículo es representativo del proceso de expansión al que aludimos al inicio de esta introducción. En un contexto de desarrollo rotundo de los estudios sobre la última dictadura cívico-militar, las miradas ancladas en espacios locales lograron no sólo visibilizar actores, tramas sociales y lógicas políticas que las perspectivas "macro", otrora dominantes, no lograban aprehender, sino también dislocar las cronologías, agendas e interpretaciones sobre este período nodal de la Argentina reciente. A través de una cartografía rigurosa y detallada de los estudios localizados sobre el funcionamiento del régimen militar y la represión - pero también atenta a los comportamientos sociales frente a su despliegueÁguila revela las potencialidades que comporta el juego de escalas a la hora de reconfigurar nuestros cuestionarios de investigación. La localización ofrece, en este caso, un punto de mira privilegiado para reponer las múltiples lógicas de actuación del poder autoritario, el desarrollo de dispositivos de violencia estatal y paraestatal, así como las formas de interacción que el régimen cultivó con los actores locales. Se trata, por cierto, de una pieza fundamental para rearmar el entramado de relaciones entre la dictadura y la sociedad, interrogante que sigue estimulando la producción sobre esta etapa. Lo municipal, recorte espacial predominante en las miradas hilvanadas por la autora, proporciona un laboratorio privilegiado 
para reflexionar sobre la "micropolítica" de la dictadura plasmada en el reclutamiento de funcionarios, en las alianzas entabladas con ciertos sectores y actores de la sociedad civil y en el papel de los medios de comunicación en los procesos de legitimación que el régimen cosechó en sede local. El acceso a la fuentes es un desafío en el que la autora repara de manera contundente, repasando la diversidad de repositorios existentes imprescindibles e inestables, por cierto- para encarar el estudio de este complejo pasado. Como se desprende de la propuesta de Águila, lejos de una perspectiva parroquial o localista, los análisis sobre la dictadura militar argentina recuperan un problema común a los procesos de implantación de los poderes autoritarios a nivel continental o global.

El cruce entre lo particular y lo global, así como la potencia explicativa de lo local a la hora de reponer procesos asociados a la historia reciente de la clase trabajadora, modelan el aporte de Mirta Lobato titulado "Lo que el capital de llevó: fábricas cerradas, memoria e historia". Se narran allí las dramáticas consecuencias del colapso y crisis productiva de la comunidad de Berisso, signada por un proceso de desindustrialización que provocó el cierre de los frigoríficos Armour y Swift y la cooperativización de la hilandería The Pattent Knitting Co, establecimientos fabriles trascendentales para el vida cotidiana y el desarrollo colectivo de dicha localidad. La crisis y desarticulación comunitaria derivada de la clausura de estas firmas, constituyen una faceta inescindible de las cambiantes dinámicas de la economía capitalista a lo largo del planeta. Los puentes que el texto de Lobato invita a trazar con otras experiencias son, en ese sentido, sugerentes. En la localidad analizada, la reflexión en torno a lo que el capital "se llevó" funciona como un disparador para reponer las implicancias del cierre de estas firmas en términos de evocación, emociones y construcción de memorias colectivas que se sostienen en diversas iniciativas colectivas. Su relato recupera y cede la palabra a múltiples actores locales -historiadores, documentalistas, asociaciones comunitarias, vecinas y vecinos- como una oportunidad para acceder a las emociones o los afectos, una dimensión trascendental de las relaciones sociales de una comunidad pasible de ser además, abordada e historizada a partir de estrategias propias del quehacer historiográfico. Su texto nos muestra también de qué manera el involucramiento de una historiadora con un lugar supone incorporar tanto su propia interpretación como las formas en que una comunidad se piensa a sí misma, arista donde cobran relevancia los historiadores no académicos, los documentalistas, los centros vecinales y tantos otros actores comunitarios capaces no solo de proveer insumos para nuestras propias investigaciones sino también de integrarse como parte activa en la construcción de las interpretaciones.

Entendemos que los balances que estos artículos ofrecen así como las propuestas metodológicas y teóricas que las autoras despliegan a propósito de los temas, problemas y contextos que abordan, sintetizan algunas de las principales apuestas de la historia en clave local. También brindan un conjunto de estrategias destinadas a establecer conexiones entre diversas escalas, que siguen las pistas de nuestros sujetos de análisis con sus intervenciones y su capacidad de acción en la delimitación de los espacios. Vistas en conjunto, las indagaciones de Susana Bandieri, Gabriela Águila y Mirta Lobato logran poner en evidencia no solo la potencia de las interpretaciones situadas en escala local, sino también valiosos rumbos para reflexionar con más densidad sobre sus perímetros.

\section{BibLiografía}

Andújar, A. y Lichtmajer, L. (Comp.). (2019). Lo local en debate. Abordajes desde la historia social, politica y los estudios de género (Argentina 1900-1960). Buenos Aires: Teseo. Recuperado de: https://www.editorialteseo.com/archi vos/16955/lo-local-en-debate/

Bandieri, S. (1996). Entre lo micro y lo macro: la historia regional. Síntesis de una experiencia. Entrepasados, VI (11), 71-100. Recuperado de https://ahira.com.ar/ejemplares/entrepasados-no-11/

Bandieri, S. y Fernández, S. (Ed.). (2017). La historia argentina en perspectiva local y regional. Nuevas miradas para viejos problemas. Buenos Aires: Teseo. 
Barriera, D. (2005). Escalas de observación y prácticas historiográficas. La construcción de horizontes alternativos de investigación. En G. Dalla Corte, et. al., Homogeneidad, diferencia y exclusión en América Latina. X EncuentroDebate América Latina ayer y hoy. Barcelona: Universitat de Barcelona.

Bohoslavsky, E. (Coord.). (2018). Dossier: Debates y conflictos de la historia regional en la Argentina actual. Quinto Sol, 22 (3), 1-51. https://doi.org/10.19137/qs.v22i3.3337

Bragoni, B. (Comp.). (2004). Microanálisis. Ensayos de historiografía argentina. Buenos Aires: Prometeo.

Carbonari, M. R. y Carini, G. (Comp.). (2020). Historia local y regional. Balances y agenda de una perspectiva historiográfica. Río Cuarto: UniRío Editora. Recuperado de: http://www.unirioeditora.com.ar/producto/histo ria-local-regional/

Fernández, S. (Comp.). (2007). Más allá del territorio. La historia regional y local como problema. Discusiones, balances $y$ proyecciones. Rosario: Prohistoria.

Fernández, S, y Dalla Corte, G. (Comp.). (2001). Lugares para la historia. Espacio, historia regional e historia local en los estudios contemporáneos. Rosario: Universidad Nacional de Rosario.

Girbal, N. (2010). La historia regional argentina en tiempos del Bicentenario de la Revolución de Mayo de 1810. Mundo Agrario, 10 (20), 1-21. Recuperado de: http://sedici.unlp.edu.ar/handle/10915/13468

Kindgard, A. (2011). Sobre historia regional y microhistoria italiana. Diálogos a propósito de una experiencia política local en la Argentina de 1930. HiSTOReLo. Revista de Historia Regional y Local, 3 (5), 113-146. https://doi.o rg/10.15446/historelo.v3n5.20654

Leoni, M. S. (2013). Treinta años de historiografía política regional. PolHis, Revista Bibliográfica Del Programa Interuniversitario De Historia Política, 6 (12), 46-53. Recuperado de: https://dialnet.unirioja.es/descarga/artic ulo/4739333.pdf

Lobato, M. Z. (2007). Niveles y dimensiones de análisis en el mundo del trabajo: notas a partir de una experiencia de investigación. Anuario IEHS, 22, 401-422. Recuperado de: http://anuarioiehs.unicen.edu.ar/resumenes/2007/14\%20Niveles\%20y\%20dimensiones\%20de\%20an\%C 3\%A1lisis\%20en\%20el\%20mundo\%20del\%20trabajo\%20notas\%20a\%20partir\%20de\%20una\%20experienci a\%20de\%20investigaci\%C3\%B3n..html

Man, R. (2013). La microhistoria como referente teórico-metodológico. Un recorrido por sus vertientes y debates conceptuales. Historia Actual Online, 30, 167-173. Recuperado de: https://dialnet.unirioja.es/descarga/articul o/4198158.pdf

Massey, D. (1995). Places and Their Pasts. History Workshop Journal, 39, 182-192.

Serna, J. y Pons, A. (2007). Más cerca, más denso. La historia local y sus metáforas. En Fernández, S. (Comp.). Más allá del territorio. La historia regional y local como problema. Discusiones, balances y proyecciones, 17-30. Rosario: Prohistoria.

Richard-Jorba, R. y Bonaudo, M. (Coord.). (2016). Historia regional: Enfoques y articulaciones para complejizar una historia nacional. La Plata: Universidad Nacional de La Plata. Recuperado de: https://libros.fahce.unlp.edu.ar/ index.php/libros/catalog/book/9

Solís Carnicer, M. (2015). La Historia Regional en la Argentina. Sus aportes y desafíos para el futuro. Folia Histórica del Nordeste, 24, 165-168. https://doi.org/10.30972/fhn.024306

Terradas i Saborit, I. (2001). La Historia de las Estructuras y la Historia de la Vida. Reflexiones sobre las Formas de Relacionar la Historia Local y la Historia General. En S. Fernández, y G. Dalla Corte (Comp.). Lugares para la historia. Espacio, historia regional e historia local en los estudios contemporáneos. Rosario: Universidad Nacional de Rosario.

Torre, A. (2018). Micro/macro: ¿local/global? El problema de la localidad en una historia espacializada. Historia Critica, 69, 37-67. https://doi.org/10.7440/histcrit69.2018.03 


\section{Notas}

1 Las reflexiones historiográficas sobre el tema se plasmaron en una larga saga de publicaciones. Véase, entre otros, Bandieri (1996); Fernández y Dalla Corte (2001); Bragoni (2004), Barriera (2005); Fernández (2007); Lobato (2007); Girbal (2010); Kindgard (2011); Leoni (2013); Man (2013); Richard-Jorba y Bonaudo (2016); Solís Carnicer (2015); Bandieri y Fernández (2017), Bohoslavsky (2018); Carbonari y Carini (2020).

2 En esa dirección puede enmarcarse la realización del Primer Taller de Investigación "Los estudios locales en perspectiva. Aproximaciones desde la historia social y política, y los estudios de género (Argentina, primera mitad del siglo XX)", llevado a cabo en noviembre de 2018 en la Ciudad Autónoma de Buenos Aires, cuyos resultados se publicaron en Andújar y Lichtmajer (2019). 